\title{
Research on Characteristics and Reasons of Current Internet Group Events
}

\author{
Lei Yang \\ Department of Journalism,School of Humanities and \\ Law \\ Northeastern University,NEU \\ Shenyang, Liaoning Province, China \\ Yanglei_ly@aliyun.com
}

\author{
Haotong Yang \\ Japanese Department;Foreign Studies \\ College;International Economic Law,Continuing \\ Education College \\ Northeastern University,NEU \\ Shenyang, Liaoning Province, China \\ Yanghaotong.yht@gmail.com
}

\begin{abstract}
Department of media management and Scholars have focused on Internet group events. This paper begins with analyzing the recently internet group events caused by hot issues in China. Through analyzing the characteristics,and causes of the Internet group events, we further clarify the basic understanding of Internet group events, which is the foundation of our further research.
\end{abstract}

Keywords- internet group events; characteristics; causes

\section{INTRODUCTION}

As we all know, the network brings the profound evolution of both information dissemination techniques and Internet audient mode compared to traditional medium audient feedback mode., Network audience express the views of network information through the network media when facing with heated social issues, this information will be of more concentrated. In theworld of network, the remarks of an ordinary Internet user, are likely to cause " one calls and million response". Moreover, remarks aggregation sometimes turn into mass incidents on the network, which have considerable impacts on both network society and realistic society.For example, in recent years the "Meimei Guo incident", "little Yue event", " Jiaxin Yao murder" and otherheated social issues have caused great disturbances in the network, forming a typical "Internet group events".

\section{THE DEFINITION OF INTERNET GROUP EVENTS}

Group events refers to the events, which is initiated by a group of public with a certain scale and purpose, causing farreaching impact on the management of government and society, such as collective petitioning, assembly, parade, blocking traffic, containment of Party and government organs, kept a vigil, riot, etc. Four criteria are used for identifying group incidents: firstly, More than five people. The statistics from related departments of group events first determine the order number, such as the Petition Regulations expressly provides as "events" for more than five people; Secondly, there is a common behavior direction; Thirdly, lacking of statutory basis on the the procedures. The last, hindering the rule, in particular, property, security and order, control order.

Group events takes place not only in the real world, but also on the network, namely Internet group incidents, hereinafter referred to as the Internet group events which indicate the discussion of hot issues participated in by Internet users.. Against the backdrop of increasingly maturing network media and sensitive stage of society development, the Internet user keep focus on strong power,public welfare, high attention "three departments"and staff,which probably lead to Internet group incidents.

\section{THE TYPES OF INTERNET GROUP EVENT}

\section{A. Internet Group Events Caused by News Public Relations}

In this kind of group events, outburst events gain the attention of netizens in a short period of time., The whirling official news cause heat to discuss and consideration for people's own interests. As the "Word of the Year 2009", "Duo Mao Mao" is the best interpretation of this kind of group events. The cause of "Duo Mao Mao" event is the death of a prisoner in Yunnan Jinning County Detention Center.. According to local police officials, 24 year old man Li Qiaoming had a head injury while playing "Duo Mao Mao" with his inmates in the detention room. After rushed to hospital for salvage, Li died despite efforts.. Such news released from official press raised doubts in public.. For a time, with the spread of suspicion and negative voice on Internet, the pursuit of truth gained attention of the local government. In the end, the truth, came out. The involved criminal suspects and government officials of malfeasance are subject to legal sanctions. The concealment and coverage of malignant event was imprilled in the developing network environment, irresponsible news network network Public Relations is the trigger to, False news and information make the Internet users feel that fairness and justice can not be guaranteed, so the Internet userstake the network as the medium to express their inner dissatisfaction.

B. Secondly, is the Network Groups Events Triggered by the Sensitive Character and Social Hotspot

2010 suffered hot Xi'an Conservatory of Music junior Yao Jiaxin car wounding at the beginning of the incident, Jiaxin Yao was about "two rich generation," "officer the second generation", "army of two generation" false title, this title is often socially sensitive vocabulary, often considered social unjusty, gradually widening gap between rich and poor in one embodiment. So in the course of the trial drug Xin cases, the masses will not help a subjective comment. Yao Jiaxin case, regardless of its nature does not talk, single 
was initially for his ID guess is enough to lift a great disturbance in the network, discontent, in reality society under pressure and can not be expressed, so with the aid of the network virtual instrument to achieve their purpose. Group events in the hot topic, surrounded by the emerge as the times require sensitive vocabulary.

\section{Thirdly, is Network Mass Incidents Caused by Internet Users Protest Policies or Measures}

Because modern society network media developed, not only a lot of government or media in the network to establish official website issued a new policy or national or local news, in the implementation of the new policy will first try on the network to listen to the netizens' opinions and suggestions. Users on the official website, web forums and other interactive network platform, in the same or opposite views on the internet widely discussed. The micro-blog real-name system was also discussed on the topic. December 16, 2011, Beijing Municipal People's Government Information Office, the Municipal Public Security Bureau, Municipal Communication Management Bureau and Internet Information Office jointly developed the "Beijing City microblogging development management a number of provisions" issued, Twitter users must be real identity information registration, in order to use speech function. This policy is not implemented, the netizen public opinions are divergent. Some people think that the network supervision, be imperative, also somebody thinks the network real-name system implementation will bring certain degree of speech restrictions, allowing Internet users no longer dare to speak the truth. Various voices together bound to rub out network group event sparks.

\section{The Last, is the Network Groups Events Triggered By the Network Culture Camp's Rebuttal To the Existing Cultural System}

Culture is also a power system, this system often lies in which the elite and powerful class. Network media, just to break the cultural power of this traditional social stratification. Internet users can also plays an important role in the online world as a power main.

\section{THE CAUSES OF INTERNET GROUP EVENTS}

\section{A. Hot Figures and Hot Events}

With the vigorous development of Internet technology, online media has naturally become an important channel for the audience to get news and information. Online media information, timeliness, promptness, convenience features, making the audience the first time to learn the latest information. In such an important premise and background, some touch of social hot topics of the characters and events occur, will be in the network caused a great disturbance. Many people often in real life under certain public opinion and the reality of the pressure, they because of such pressure could not be openly expressing their true inner thoughts, and the network of heterogeneous platform, just give these people bring to express comments, comments the important opportunity. There are even quite one part of speech and thought more extreme users, because some sensitive topic talk a lot of nonsense, instigated the masses to reactionary mood, causes a netizen to a events were "lookers", "irrigation", make the network group events in reality interpretation have bedding.

2011"Jiaxin Yao car murder", Xi'an Conservatory of Music junior Jiaxin Yao short time caused considerable concern. With the further investigation of the case, the parties of the background is also gradually reveal to the public, it immediately in the online form two camps, some people strongly condemn Yao arrogant behavior, others with a sympathetic attitude, two diametrically opposed views on the network caused controversy, once the trial brought negative effects.

\section{B. Information Channel is not Open}

The network group event is a collection behavior. "Collection behavior of the main forms of information is a rumor. Gossip is a source of unknown, can not be confirmed the news or speech, usually occurs in the social environment with higher uncertainty, and formal communication channels (such as mass media) is not smooth or function period. Gossip has two characteristics: first, gossip is usually around more relationship problems, involving the vital interests of important problems occurred; second, from the formal channels of the evidence insufficient information, status of ambiguity increases, will drive people to seek information by rumor channel." Network group incidents had occurred, from the users based on a kind of uncertainty arising from guessing the nature of speech. This uncertainty is because the official media or government departments in many emergency event to give the public a timely, objective interpretation, the netizen curiosity rising, a lot of radical friends will inevitably take this opportunity to spread rumors to create trouble, leading to circulate erroneous reports, false, exaggerated information grow strong, evoked group events, network society and the realistic society to bring certain adverse effects.

In the summer of 2011"7• 23 Wenzhou motor car accident", Once again to verify the information channel is not smooth for network groups events catalysis. Since the incident in the middle of the night, so a lot of Internet users in the first time to understand it is not official news, but by the passengers on the network of microblogging. The next day, the official news blots out the sky, but the coverage of the key content is nothing but the rescue work situation, casualties and aftercare work. In the face of such bad car crash, the situation is very fierce, many users can send the accident of the so-called "real casualties" as well as the cause of the accident is. The number of days, the railway ministry held a press conference to announce the recent casualties, but did not give a formal interpretation of the cause of the accident. Official information limited, naturally brought friends endless speculation psychological, and even a lot of friends through television and Internet video shots, confirmed his suspicion of the casualties, criticized the Ministry of Railways various improper handling. No official information on the positive response, such remarks because 
some blurry screenshots and recording "evidence" and it shows certain persuasion, attracted a lot of users and professionals, on the network in response to the accident are discussed more fierce. Therefore, the lag induced by rumors of official information, rumors, is also an important reason for the network group events.

\section{The network of speech needs of audience}

Under the environment of the online medium, the audience is no longer simply receive the information by the identity of any person can become the main body of information dissemination, people can also spread the news, in a speech at the same time, be recognized by others, cause resonance, realize self value. Maslow's hierarchy of needs theory points out, with respect to the needs and self actualization need is high need, people all hope to get stable social status, get social respect and recognition. The speech needs, hope to get more recognition and respect. If things go on like this, people accustomed to receiving news also can say something, formed the habit of thoughts. In this idea and the role of demand, a lot of people spontaneously in the same event, the same news interactive discussion. The more sensitive the topic, the more you attract more network audience to join the discussion, the network groups events also emerged.

\section{THE CHARACTERISTICS OF INTERNET GROUP EVENTS}

\section{A. Criticism of the Reality Profoundly}

In the real world, a lot of people on socially sensitive topics and hot figures discontent is difficult to express the true inner thoughts only through the anonymous way to say. Or, it is people have a real problem doubts and investigate, let a lot of the dark side of society proud disclosure. The network presented by the social reality and the sunny side opposite side will cause the attention and participation, onlookers were growing, vent their dissatisfaction.

Review the events of recent years, from the "most cattle nail house" to the "tiger week", from "my dad is Li Gang" Jocie Guo "to flaunt wealth", is not difficult to find such events as well as its protagonist identity involving social groups, mostly and officials, merchants, officials, two generation two rich generation and corruption phenomenon is closely related to. Ordinary people for the "money power groups" doubts exist, on social vulnerable groups sympathetic to their vital interests, and pay close attention to very, once these problems together, cause masses emotional excitement, on the network outlet to protest the mood, criticism of social reality, in order to get wide attention of social existence, fantasy the contradiction can be solved properly.

\section{B. Strong Group Polarization}

The concept of group polarization originated from the University of Chicago Law School Professor Sunstein's "network of the Republic: the issue of democracy in the network society". In his book: "group polarization definition and simple, group member begins with some bias, in deliberation, the people towards bias in the direction to move, the final formation of extreme views." In addition, his research has also pointed out, the netizen "polarization" tendency is more prominent. This is not difficult to understand, a risk factor for both network groups incident is also an important feature.

Internet users as a large group, is substantially different from the reality of the group. Realistic group expression are often subjected to identity, status, knowledge level constraints, in addition to the pressure of public opinion and population pressure, can not act recklessly and care for nobody's telling the truth and reality of social people in a society live characteristic. Network world and the real society is substantially opposite, on the Internet say more freedom and go all lengths, Some users even have the "angry youth" mentality, the "reform package", the potential in the heart of the original impulse to be released in full, setting off the waves of public opinion of the network society.

\section{Ambiguous And the Reality Interactive}

Although the network of people and the reality of human behavior are very different, but the two are not completely out of the opposite. .The network of speech on the Internet is actually the real social phenomenon of the reaction, is more or less have some basis in reality. When a thing is caused on the network the majority of Internet users to resonate, causes the network group events, the majority of users emotional nowhere escape, will in reality from triggering mass incidents. Then the opinion of effects brought about by the network of "virtual" interaction between the reality of the "real" will show up.

Popular in recent years on the network "human flesh", is a vivid combination of the online world and social reality examples. The so-called "human flesh search", is the use of modern information technology (computer, TV, radio, newspaper and so on), the network changed the traditional information search find people, people ask people, touch people, people, people who endure the relational network community activity, change dull the query process for a person to ask questions in response, eight, one stone arouses 1000 billow, calling out 10000 awoke really, make every attempt to search experience. Network group broke out after the incident, the users using network spread widely, according to the Internet known clues in the search for the real hero. Social performance thereon expose, bring certain social order disorder, for social stability and harmony to create pressure, is a fuse of social unrest.

\section{Different Speech Continuity}

In the event of network groups, continued to surge in the degree of hot topic of concern or person for attracting the audience the different levels, for an event, concern and loyalty fluctuations, leading to some event, while some events soon fade in people's attention. Network group events has such characteristics, On the one hand because of some hot events or people can only lead to attention and discussion in a short period of time, it may be properly resolved and dealt with the unfairness other reasons will be limited free speech of Internet users, the real idea too less than expression. 
With the technological and economic development, the rapid development of the Internet, an increasing role of the online medium, coincided with the social transformation of complex and diverse, contradictory, network group events showing a multiple trend. We analyze the characteristics of the network group events and causes of the network mass incidents in recent years, Paving the way for our next indepth study on the network group events for the network news ecology.

\section{ACKNOWLEDGMENT}

In the process of writing, I has learned a lot from theory predecessors, and corrected my own mind about thesis and some of my own misunderstanding of expertise, finally finished the ends of the paper. I sincerely thank my friends, who devoted their efforts and patience to each chapter and progress in every step in my thesis.

The project of Shenyang's Philosophy and Social Science special issue"Shenyang Economic Area media resources and the role of countermeasures"(0011020).

\section{REFERENCES}

[1] G. Eason, B. Noble, and I. N. Sneddon, "On certain integrals of Lipschitz-Hankel type involving products of Bessel functions," Phil. Trans. Roy. Soc. London, vol. A247, pp. 529-551, April 1955. (references)

[2] J. Clerk Maxwell, A Treatise on Electricity and Magnetism, 3rd ed., vol. 2. Oxford: Clarendon, 1892, pp.68-73.

[3] I. S. Jacobs and C. P. Bean, "Fine particles, thin films and exchange anisotropy," in Magnetism, vol. III, G. T. Rado and H. Suhl, Eds. New York: Academic, 1963, pp. 271-350.

[4] K. Elissa, "Title of paper if known," unpublished.

[5] R. Nicole, "Title of paper with only first word capitalized," J. Name Stand. Abbrev., in press.

[6] Y. Yorozu, M. Hirano, K. Oka, and Y. Tagawa, "Electron spectroscopy studies on magneto-optical media and plastic substrate interface,” IEEE Transl. J. Magn. Japan, vol. 2, pp. 740-741, August 1987 [Digests 9th Annual Conf. Magnetics Japan, p. 301, 1982].

[7] M. Young, The Technical Writer's Handbook. Mill Valley, CA: University Science, 1989. 